\title{
The effects of urine level, duration of treatment and moisture level on nutritive value of wheat straw
}

\author{
VAP Cadavez, AA Dias-Da-Silva \\ Department of Animal Production, Universidade de Trás-OS-Montes e Alto Douro 5000 Vila Real - Portugal
}

The utilization of urine as a source of ammonia for upgrading the nutritive value of wheat straw was investigated. Two experiments arranged in a factorial design $(3 \times 3)$ with 3 replicates per treatment were performed using the same batch of wheat straw. In Experiment 1 moisture level was fixed adding water to the straw when necessary. In Experiment 2 moisture level changed according to the amount of urine applied. The levels of urine applied $(20.4 \mathrm{~g}$ urea per litre) in both experiments were 100 (U1), 200 (U2) or $300 \mathrm{ml}$ (U3) per $100 \mathrm{~g}$ straw. For control, $100 \mathrm{~g}$ straw samples were treated with $50 \mathrm{ml}$ urea solution (120 g urea per litre). Prior to treatment straw was coarsely chopped $(4 \mathrm{~cm})$. All samples were stored in sealed plastic bags at $25^{\circ} \mathrm{C}$ for 3,6 or 9 weeks.

The criteria reported in this paper to evaluate the upgrading effects were neutral detergent fibre (NDF) and dry matter loss (DML) after 72 $h$ incubation in the rumen. The main results are shown in the Table.

The results indicate that for low levels of urine/urea application (U1 and U2) the upgrading effect is small and decreases with time particularly if urine is diluted. When applied at the highest level, urine treatment increased DML over untreated straw ca 100 $\mathrm{g} / \mathrm{kg}$. Although the amount of residual urea was less than $1 \%$ of the urea applied as urine in all cases, the best response to urine treatment in terms of DML remains below the response obtained with the "standard" treatment with urea solution which was 664, 707 and 717 $\mathrm{g} / \mathrm{kg}$ after 3,6 and 9 weeks of treatment, respectively.

Effects of urine treatment on NDF and DML of wheat straw ( $\mathrm{g} / \mathrm{kg}$ straw dry matter)

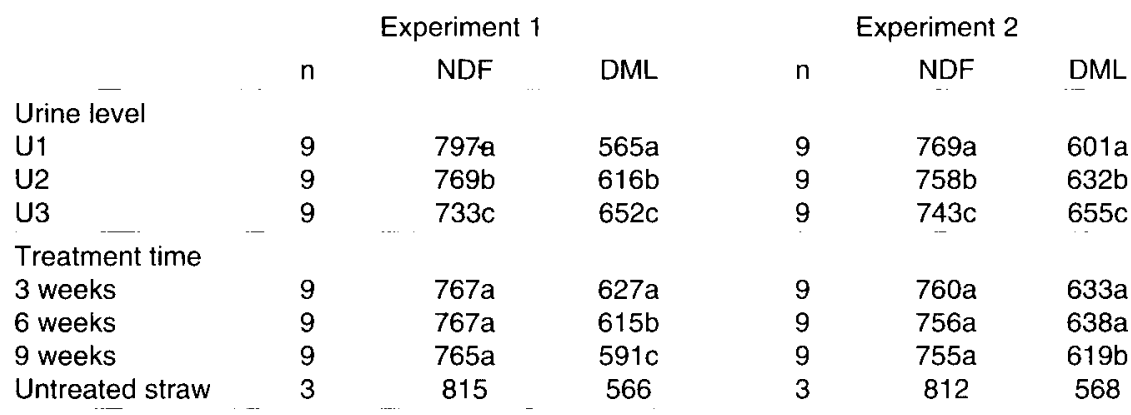

Means with different superscripts within a column for each main factor are significantly different $(P<0.05)$. 